\title{
The measurement of low impulse from pulsed electromagnetic thrusters by thrust stand
}

\author{
Chenyang Ding ${ }^{1, a}$, Mousen Cheng ${ }^{1, b}$ \\ ${ }^{1}$ School of Aerospace Science and Engineering, National University of Defense Technology, \\ Changsha 410073, China; \\ a15616453882@163.com, bmscheng@nudt.edu.cn
}

Keywords: Low impulse measurement, thrust stand, pulsed electromagnetic thruster.

\begin{abstract}
Two types of measurement methods are given to determine the low impulse involved in the working process of pulsed electromagnetic plasma thrusters. Different mechanical devices, including pendulum and critical stress thrust stands, are introduced and compared with others concomitant with respective typical applications. Advantages as well as disadvantages of these thrust stands are discussed, which can provide a favorable reference for pulsed electromagnetic plasma thrusters’ micro-impulse measurement and research.
\end{abstract}

\section{Introduction}

With the rapid development of space pulsed electromagnetic thrusters, the research of thrust measuring devices is becoming popular. Compared with the conventional electric propulsion devices, measurement of low impulse for pulsed electromagnetic thrusters may become tougher because many unique problems may occur in the process of firing, such as electromagnetic interference, zero drift and influence of environmental factors. These problems increase the uncertainty of impulse measurement and call for novel designs of mechanical structures for measuring the lower impulse and advanced instruments with higher resolution and sensitivity.

Performance evaluation is one of the essential components involved in the design of electric propulsion systems. This process, in general, is completed by precisely measuring the impulse or thrust during its working period. There are two ways to measure impulse or thrust for pulsed electromagnetic thrusters. One is using field probes [1] to measure the magnetic field and plasma density by virtue of reasonable arrangement of probes in the plasma flow field. Thrust can be determined by the integral of unit Lorentz force $(\vec{j} \times \vec{B})$ in the whole area. This approach lends the possibility to achieve the thrust instantaneously during thruster's working process and drawing the thrust curve. But the paramount disadvantage is that the results are often larger than the real ones, thus causing significant errors [2]. Another method is to use mechanical thrust stand that can directly measure the impulse of thrusters, followed by calculating the specific impulse and efficiency of thrusters. This approach is more adoptable than the former one in that it can reduce the interference of uncertain factors to a larger extent.

\section{Theory of determining the impulse}

\subsection{Equivalent performance}

One of the calibration methods to measure the impulse according to platform's response is called equivalent performance. After recording the largest displacement of the pulsed working thruster during one period, equivalent pulse can be applied on the thruster after the engine flameout to give the same performance. For example, the completely inelastic collision brought by a ball clay can be regarded as the equivalent performance by keeping the same displacement as the former.

\subsection{Analytical method}

Another approach is using the mechanical linkage in the thrust stand to reproduce or amplify the small displacement detected by the displacement sensor. According to the impulse vibration differential equation in structural dynamics: 


$$
a \ddot{y}+b \dot{y}+c y=W
$$

Where a, b, c is inertia constant, damping coefficient, stiffness coefficient respectively. And $y$ is the displacement while $\mathrm{W}$ is the calibration quality (when used in the thruster, $\mathrm{W}$ represents the pulsed thrust $F(t)$ ).

By adding the initial condition, equation (1) can be solved as follow:

$$
\dot{y}=\frac{W}{\omega a} e^{-\beta t} \sin \omega t
$$

Where $\beta=\frac{b}{2 a}, \omega=\sqrt{\frac{c}{a}-\beta^{2}}$, both of which can be obtained from the vibration curve of the thrust stand.

The velocity may peak up at $t=\frac{\tau}{4}$ ( $\tau$ is the period),

$$
\dot{y}_{\max }=\frac{W}{\omega a} e^{-\beta \frac{\tau}{4}}
$$

Then a, b, c can be solved, $a=\frac{W}{\omega \dot{y}_{\max }} e^{-\beta \frac{\tau}{4}}, b=2 a \beta, c=a\left(\omega^{2}+\beta^{2}\right)$.

Because the period of a discharge pulse tends to be quite short when compared to the vibration period of thrust stand, velocity caused by a single pulse is as follows ( $\delta$ is the time period of a discharge pulse):

$$
\dot{y}_{t=\delta}=\frac{1}{a} \int_{0}^{\delta} F(t) d t=\frac{I}{a}
$$

The first order item $b$ and zero order item c, brought by the negligible elastic and damping force, can be ignored. Therefore, the working impulse of the thrust stand can be analytically obtained as $I=a \dot{y}_{t=\delta}$.

\section{Classification and typical applications of impulse measurement}

According to the mechanical structures and the principles of thrust measurement, impulse measuring thrust stands can be classified as follows: hanging pendulum, inverted pendulum and torsional pendulum and critical stress. All these types of reasonable structures can reproduce or even amplify the small displacement after the thruster's ignition. With the signal acquired by the transducer that give the real-time displacement or velocity information, the thrust or impulse in the thruster's working process can be determined.

\subsection{Hanging pendulum}

Using gravity as a restoring force, this kind of thrust stand can achieve a high stability with the simple configuration under the external force disturbance [3]. The tested thruster generally is fixed on one side of the swing arm. When the thruster ignites to work, it will drive the swing arm to rotate, producing a small deflection that can be directly measured by a displacement transducer. Sometimes, when the displacement is too tiny to be detected, some optical means can be put into operation, such as laser interferometry.

Polzin, from NASA Marshall flight control center, developed a novel hanging pendulum thrust stand (variable amplitude hanging pendulum with extended range, vahper) [4], which could accurately measure the thrust in $1 \mathrm{mN}$ level produced by the thruster up to $125 \mathrm{~kg}$. The tiny horizontal displacement can be amplified to a vertical deflection by means of an internal low-friction mechanical linkage. By adjusting the pivot position (point D in figure 1), this stand can measure a wide range of thrust levels. With combination of the low friction pivot, the ratio non-contact displacement sensor, the effective load level adjustment and thermal control system, this system effectively eliminated the zero drift problem in the traditional pendulum thrust stand. 


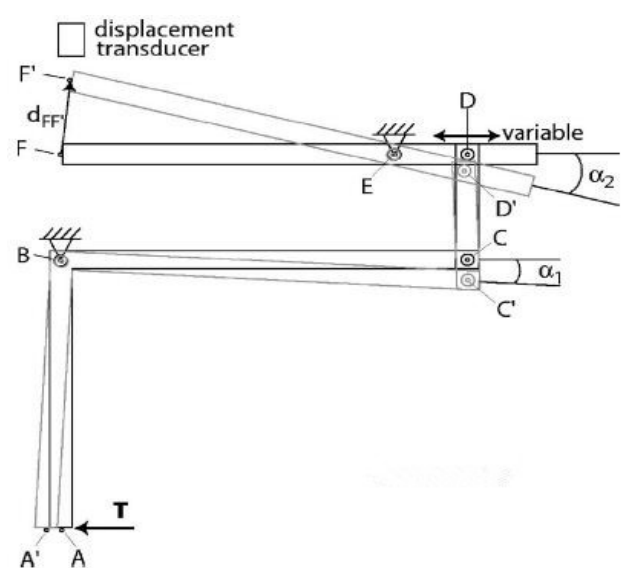

Fig. 1 Displacement transform of vahper[4]

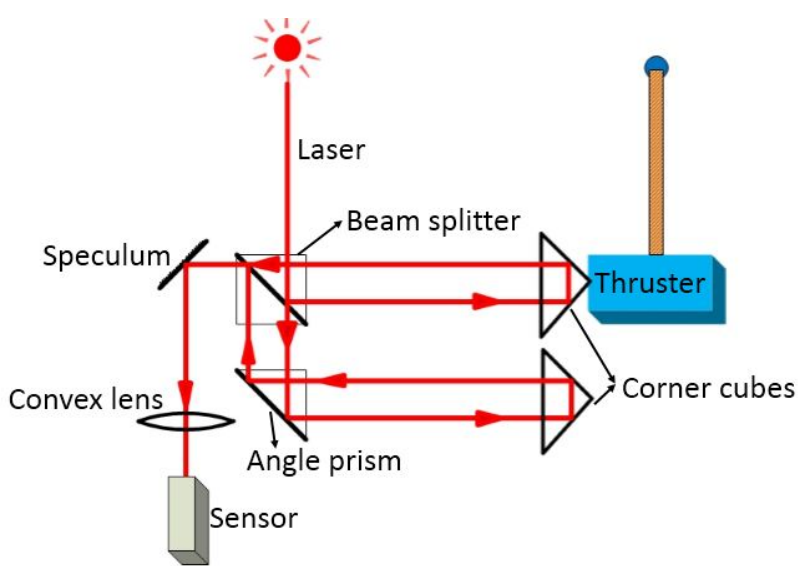

Fig. 2 Working principles of ims

Cubbin, from Princeton University, designed an interferometric measurement system (ims) to determine the pulsed thrust of electric thrusters [5]. The laser source generated a laser beam and then split into two beams, passing two angle prisms (one was fixed on the bench and another was mounted on the thruster). Due to the phase difference $\emptyset$, interference effect occurs. When the thruster fires, one of the angle prisms will move, leading the change of interference phase, by which can the impulse determined.

Based on the principle of Michelson interferometer, ims well solved the electromagnetic interference problems in the impulse measurement of high-current pulsed plasma thrusters. In addition, the resolution of the measurement system can reach up to $10 \mathrm{~nm}$ level, which inevitably brought in a larger defect that ims is only applicable to the nanoscale measurement of micro-thrusters.

\subsection{Inverted pendulum}

The tested thruster (or counterweight) is usually fixed on one side of the swing arm, above the flexural pivot [6]. In terms of structural stability, the inverted pendulum is slightly inferior to the hanging pendulum structure while the sensitivity of inverted pendulum is much higher than traditional hanging pendulum structure influenced by the pivot friction. The stability of inverted pendulum is the strong function of supporting stiffness that is influenced by environmental factors, such as temperature, thus increasing the uncertainty of measurement results, which is the major problem of inverted pendulum thrust stands.

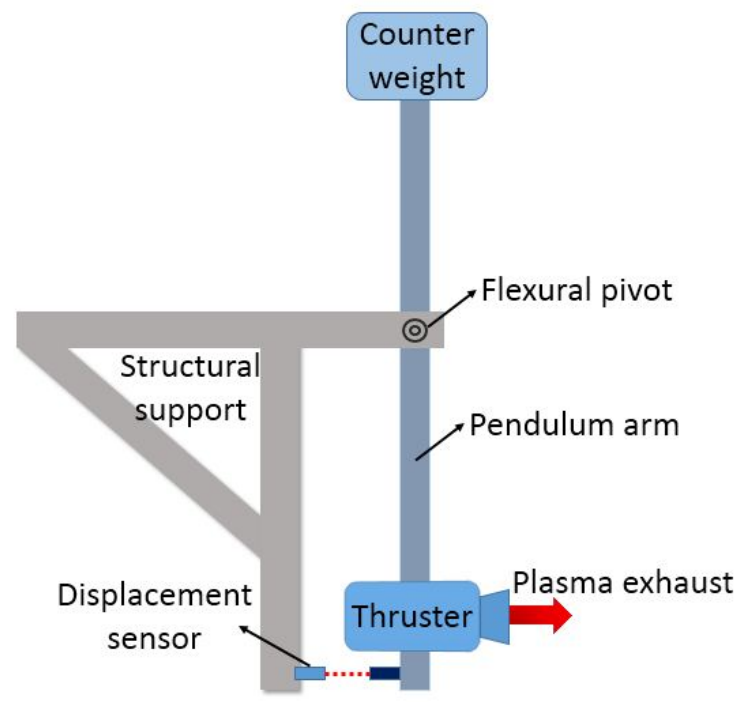

Fig. 3 Inverted pendulum thrust stand

Haag, from NASA Lewis research center, put forward a kind of inverted pendulum thrust stand [7] that can measure impulse of pulsed electromagnetic thrusters in both single and repetitive pulse working mode, keeping the measuring precision within $1 \%$. The restoring force was provided by the elastic force from supporting system. And the supporting components could also act as a conductive material and cooling line. This structure is suitable for large thrusters' impulse measurement while it 
may bring certain errors for small and medium-sized thrusters' measurement due to the influence of the propellant supply pipeline.

\subsection{Torsional pendulum}

Thrusters mounted in this configuration [8] can rotate around the axis of gravity. Its restoring force has nothing to do with the mass of tested the thruster, lending the high sensitivity of torsional pendulum thrust stand. It was often used in the measurement of micro thrust of the pulsed thruster that requires high precision. Its asymmetric horizontal layout is a big drawback, which limits the experiments in small vacuum facilities. Linear voltage displacement transducer ( $L V D T)$ can be used to measure the small deflection of the thruster. In addition, an angle sensor also can be used to measure the angle of the torsional arm.

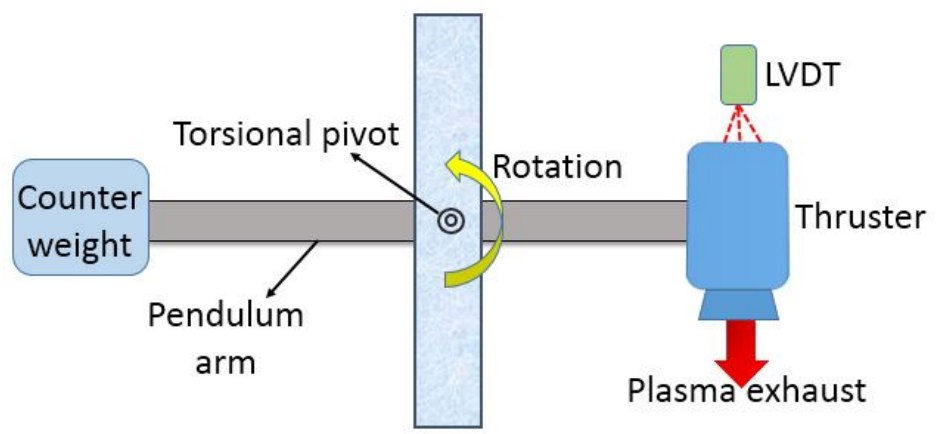

Fig. 4 Planform of torsional pendulum

Koizumi, from the University of Tokyo, developed a novel torsional pendulum thrust stand [9] to measure the impulse of liquid propellant pulsed plasma thrusters. It consists the torsional pivot, electromagnetic damper and other components. This torsional pendulum can accurately measure the impulse in $\mu \mathrm{Ns}$ level. However, it is only applicable to the micro-thrusters' impulse measurement.

\subsection{Critical stress}

It is a unique thrust measurement configuration that was originally designed for evaluating the performance of Mk II pulsed inductive plasma thrusters in 1970s [10]. After several generation of Mk pulsed inductive plasma thrusters' experiments [11, 12], the measurement technology is relatively mature. In this design, two rectangle aluminum structures are supported vertically by four flexible stainless steels. After the thruster is placed on the structure, the effective elastic coefficient of flexible supporting structure is reduced. As the mass of load increasing, the elastic coefficient decreases continuously, eventually reaching zero. At this time, the supporting structure achieves the critical elastic buckling point. A highlight of this design sits in the usage of critical elastic buckling steels, which stably support the heavy mass of the thruster as well as enhance the ability of response to external impulse. The simple configuration of this impulse measuring platform has a high sensitivity, especially suitable for low impulse measurement of pulsed thrusters with heavy mass. However, this thrust stand can only be applied to the thrusters working in a single pulse and requires strictly quiet working environment.

\section{Summary}

By comparing different types of thrust stands, it can be found that the pendulum thrust stands have a wide range of application in pulsed measurement in which the optical measurement technology is suitable for micro impulse measurement with high precision while the critical stress measuring technology can meet the need of low impulse measurement of pulsed inductive thrusters with heavy mass and large size. Since the type of thrust measurement is closely related to the certain performance of propulsion devices, it is foreseeable that the design of typical thrust measurement structures with higher sensitivity and accuracy is still the hotpot of future research. 


\section{Acknowledgments}

This work was carried out by Chenyang Ding and Mousen Cheng. We gratefully acknowledge the valuable cooperation. Chenyang Ding is the corresponding author.

\section{References}

[1] C. L. Dailey, R. H. Lovberg, Large Diameter Inductive Plasma Thrusters, Princeton/AIAA/DGLR 14th International Electric Propulsion Conference, (1979)1030.

[2] K. A. Polzin, Faraday Accelerator with Radio-frequency Assisted Discharge (FARAD), A Dissertation to the Faculty of Princeton University in Candidacy for the Degree of Doctor of Philosophy, Princeton University, 2006.

[3] T. Moeller, K. A. Polzin, Thrust Stand for Vertically Oriented Electric Propulsion Performance Evaluation, Rev. Sci. Instrum. 81, (2010)115108.

[4] K. A. Polzin, T. E. Markusic, B. J. Stanojev, et al., Thrust Stand for Electric Propulsion Performance Evaluation, Rev. Sci. Instrum. 77, (2006)105108.

[5] E. A. Cubbin, J. K. Ziemer, E. Y. Choueiri, et al., Pulsed Thrust Measurements Using Laser Interferometry, Rev. Sci. Instrum. 68, (1997)2339.

[6] K. G. Xu, M. L. R. Walker, High-power, Null-type, Inverted Pendulum Thrust Stand, Rev. Sci. Instrum. 80, (2009)055103.

[7] T. W. Haag, Thrust Stand for Pulsed Plasma Thrusters, Rev. Sci. Instrum. 68, (1997)2060.

[8] S. M. Merkowitz, P. G. Maghami, A. Sharma, et al., A $\mu$ Newton Thrust-stand for LISA, Phys. Rev. Lett. 85, (2000)2869.

[9] H. Koizumi, K. Komurasaki, Y. Arakawa, Development of Thrust Stand for Low Impulse Measurement from Microthrusters, Rev. Sci. Instrum. 75, (2004) 3185.

[10]C. L. Dailey, R. H. Lovberg, Pulsed Inductive Thruster Component Technology, AFAL TR-07-012.

[11]C. L. Dailey, R. H. Lovberg, The PIT Mk V Pulsed Inductive Thruster, NASA CR-191155.

[12]D. Russell, J. H. Poylio, W. Goldstein, et al., The Mk VI Pulsed Inductive Thruster, Space 2004 Conference and Exhibit 28-30 September 2004, San Diego, California. 